\title{
Cardiovascular Disease and COVID-19: Issues in Women
}

\author{
Sujatha Vipperlaํㅜ Anil Kumar Mahapatro ${ }^{1}$ \\ ${ }^{1}$ Department of Cardiology, Indus Hospitals, Visakhapatnam, \\ Andhra Pradesh, India
}

Ind J Car Dis Wom:2020;5:195-199

\begin{abstract}
Address for correspondence Sujatha Vipperla, MD, DM, FIC, FSCAI, Department of Cardiology, Indus Hospitals, Visakhapatnam 530002, Andhra Pradesh, India (e-mail: sujasri@hotmail.com).
\end{abstract}

\author{
Abstract \\ Keywords \\ - cardiovascular disease \\ - COVID-19 \\ - Women
}

COVID-19, although primarily a respiratory illness, has myriad cardiovascular manifestations. Underlying comorbidities like hypertension, diabetes, and underlying cardiovascular disease are associated with higher mortality. Diabetes confers greater risk in women than men. Myocardial injury is seen in 17 to $36 \%$ of patients and is associated with higher mortality. Acute coronary syndromes (ACS) pose diagnostic and therapeutic challenges in the setting of the COVID-19 pandemic. Case reports of myocarditis have been documented. The sex differences in these common presentations have not been disaggregated and such evaluation is warranted to elucidate future interventions.

\section{Introduction}

Cardiovascular involvement is reported in COVID-19 and carries a grave prognosis. The mechanisms of cardiac injury include the following:

1. Nonischemic myocardial injury: Severe form of COVID- 19 disease can lead to cytokine storm, which can result in multiorgan damage including the heart.

2. Myocardial demand supply mismatch: Increased cardiometabolic demand and hypoxia in the setting of COVID-19 infection can result in myocardial oxygen demand-supply mismatch which, in turn, leads to acute myocardial injury. ${ }^{1,2}$

3. Plaque rupture and coronary thrombosis: Viral infection is associated with a systemic inflammatory state, which creates a prothrombotic milieu that along with the increased shear stress precipitates plaque rupture, resulting in acute coronary syndrome (ACS).

4. Direct viral myocardial injury (viral myocarditis): SARS-CoV-2 binds to the membrane angiotensin-converting enzyme-2 (ACE-2) expressed in heart. The virus replicates in the myocardial cells and causes direct cell damage and activation of T lymphocytes, causing cell-mediated cytotoxicity, which is aggravated by cytokine storm syndrome. $^{3}$

5. Microvascular dysfunction: This can occur from diffuse microthrombi and vascular injury.
6. Stress-related cardiomyopathy: Takotsubo syndrome can cause left ventricular (LV) dysfunction.

7. Various therapies-related adverse effects: Antiviral drugs, corticosteroids (used in higher doses), and other therapies can have deleterious effects on the cardiovascular system. Myocardial injury, electrolyte imbalance from critical illness can precipitate arrhythmia.

\section{Cardiovascular Comorbidities and COVID-19 Infection}

Hypertension, diabetes and cardiovascular disease are prevalent among patients with COVID-19 infection and are associated with higher mortality. Wu et $\mathrm{al}_{1}{ }^{4}$ in an analysis of 18465 patients with confirmed COVID-19 infection, studied the sex-specific differences in the risk of cardiovascular morbidity and COVID-19 mortality. The median age of patient population was 50.5 years and included $47.8 \%$ women. As much as $13.87 \%$ had hypertension, $5.53 \%$ had diabetes, and $4.45 \%$ had cerebrovascular disease, $2.51 \%$ had lung disease, and $0.81 \%$ had chronic liver diseases. Compared with women, men were more likely to have at least one comorbidity (27.37\% vs. $24.98 \%$; $p<0.001)$. The prevalence of diabetes (5.81\% vs. $5.23 \% ; p 0.092$ ) and cerebrovascular diseases (CBVDs) ( $4.66 \%$ vs. $4.23 \% ; p$ 0.16) were similar in men and women. Hypertension (14.73\% vs. $12.92 \% ; p<0.001)$, lung diseases $(2.92 \%$ vs. $2.06 \% p<0.001)$, chronic kidney 
diseases ( $0.94 \%$ vs. $0.66 \% ; p 0.036$ ), and chronic liver diseases ( $0.84 \%$ vs. $0.49 \%$; $p 0.004$ ) were more prevalent in men than in women. Mortality was significantly higher in patients with hypertension (hazard ratio [HR] 1.55, 95\% confidence interval [CI] 1.35-1.78), diabetes (HR 1.35, 95\% CI 1.13-1.62), CBVDs (HR 1.70, 95\% CI 1.43-2.02), chronic kidney diseases (HR 2.09, 95\% CI 1.47-2.98), and at least two comorbidities (HR 1.84, 95\% CI 1.55-2.18) after adjusting for age, gender, and severity of illness. Women with diabetes had a higher mortality than men (HR 1.69, 95\% CI 1.27-2.25).

\section{Acute Myocardial Injury and Myocarditis in COVID-19}

Cardiovascular involvement can occur at any stage of the COVID-19 infection. Myocardial injury is defined as elevation of cardiac troponin above 99th percentile of upper reference limit. In a retrospective cohort study, Zhou et $\mathrm{al}^{5}$ analyzed the risk factors for mortality in adult hospitalized patients with COVID-19. Older age, d-dimer greater than $1 \mu \mathrm{g} / \mathrm{mL}$, and elevated troponin I were associated with higher mortality. They also found elevated troponin I in 46\% of nonsurvivors versus $1 \%$ in survivors. In an analysis conducted by Shi et $\mathrm{al}^{6}$ in hospitalized patients, $50.7 \%$ of whom were women with a mean age of 64 years, $19.7 \%$ had cardiac injury. The same group ${ }^{7}$ analyzed 671 eligible hospitalized patients with severe COVID-19, with a median age of 63 years and included $52 \%$ women, of whom $9.2 \%$ died and more often had myocardial injury (75.8\% vs. 9.7\%; $p<0.001$ ) than survivors. They reported that mortality was $50 \%$ (53 of 106) for patients with a cardiac troponin I (cTnI) $>0.026 \mathrm{ng} / \mathrm{mL}$ compared with just nine deaths (1.6\%) among 565 patients with a lower cTnI. They found no difference in incidence of myocardial injury between men and women. The patients who died were older and more often male than the survivors $(p<0.001)$. Prevalence of hypertension, diabetes, coronary heart disease, chronic renal failure, chronic heart disease, and CBVDs was higher among the patients who died. Lala et $\mathrm{al}^{8}$ analyzed 2,736 patients with COVID-19 infection admitted to five hospitals in New York, of whom 36\% patients had elevated troponin I levels within 24 hours of admission. They concluded that even mild elevation of troponin I levels portended an increased risk of death (HR 1.75, 95\% CI 1.37-2.24, $p<0.001$ ) and this risk was tripled in patients with a marked elevation in troponin I levels (HR 3.03, 95\% CI 2.42-3.80, $p<0.001$ ) (-Table 1).

\section{Acute Coronary Syndromes (ACS)}

The proinflammatory state in COVID-19 infection triggers ACS by destabilizing plaques which could be further aggravated by the presence of a prothrombotic milieu in the setting of an infection. The largest database of ACS in India is the Kerala ACS registry (only $22.8 \%$ women), in which ST-segment elevation myocardial infarction (STEMI) constituted $40 \%$ of all ACS patients, with a high mortality rate of $8.2 \%$ in the hospital. Delays in presentation were common, with $41 \%$ of STEMI presenting more than 6 hours after onset of pain. Fibrinolytic therapy was administered in $41 \%$ of STEMI patients. ${ }^{9}$ It is accepted that women with ACS have atypical presentations and undergo less aggressive diagnostic and therapeutic measures. There is paucity of data in India to ascertain the gender differences. The current pandemic poses additional challenges in the management of ACS due to further delays in presentation, nonavailability of rapid tests for SARSCoV-2 which in effect have low sensitivity, lack of personal protection equipment (PPE), and the confounding effect of mild troponin elevations observed in SARS-CoV-2 infection. Numerous studies ${ }^{5-8}$ found troponin elevation in COVID-19, which qualifies as non-ST elevation myocardial infarction and is associated with increased mortality. Most of these patients were managed conservatively and the incidence of coronary artery disease (CAD) was lacking in these studies.

In a case series ${ }^{10}$ of 18 patients with COVID-19 who had STEMI (17\% women), 10 patients (56\%) had ST elevation at admission and another 8 developed ST elevation during hospitalization (median 6 days). Only 33\% had chest pain at admission. Fourteen (78\%) patients had focal ST elevation. Nine patients (50\%) underwent coronary angiography, 6 of these patients (67\%) had obstructive disease, and 5 underwent percutaneous coronary intervention (1 after fibrinolysis). Patients with myocardial infarction had higher peak troponin and D-dimer levels than the patients with noncoronary myocardial injury. Thirteen patients (72\%) died, of whom 4 had myocardial infarction (50\%) and 9 sustained noncoronary myocardial injury (90\%). This case series reported a high incidence of noncoronary myocardial injury, which was paradoxically associated with a very high mortality of $90 \%$.

Chieffo $^{11}$ concluded from a primary analysis performed in Lombardy on 33 patients with COVID-19, who underwent urgent coronary angiography (90.9\%) or coronary computed tomography angiography (CCTA) (9.1\%), that $60.6 \%$ of these patients did not have a culprit lesion requiring treatment. Stefanini et al $^{12}$ in a retrospective analysis on 28 COVID-19 patients (28.6\% women), who were admitted for STEMI, reported a high incidence (39.3\%) of nonobstructive CAD in 11 patients. The status of SARSCoV-2 test was unknown at the time of coronary angiography in 24 patients (85.7\%). STEMI could be the first clinical presentation of COVID-19 and a large proportion had nonobstructive $\mathrm{CAD}$, which could be attributable to a type 2 myocardial infarction, endothelial dysfunction, or a cytokine storm. In another multicenter retrospective study, ${ }^{13}$ including 78 patients (63\% men), with a median age of 65 years, 59 patients (76\%) received fibrinolytic therapy and only 19 patients (24\%) were treated with primary percutaneous coronary intervention (PCI). Adverse outcomes in the form of intubation and cardiac resuscitation occurred in 16 patients (84\%) treated with primary PCI. As much as $21 \%$ had stent thrombosis, which was unacceptably high, and 5 patients (26\%) died. Fibrinolytic therapy was successful in 50 patients (85\%). Hemorrhagic stroke occurred in 5 patients (9\%). Adverse outcomes in the form of intubation and cardiac resuscitation occurred in 11 patients (19\%) and death in 4 patients (7\%). A high rate of stent thrombosis was 
Table 1 Myocardial injury in various studies

\begin{tabular}{|c|c|c|c|c|}
\hline Study & $n$ women \% & Myocardial injury & ACS & In-hospital mortality \\
\hline Zhou et $\mathrm{al}^{5}$ & $\begin{array}{l}191 \\
38 \%\end{array}$ & $17 \%$ & Autopsy in one consistent with MI & $\begin{array}{l}28.3 \% \\
\text { With cardiac injury 59\% }\end{array}$ \\
\hline Shi et $\mathrm{al}^{6}$ & $\begin{array}{l}416 \\
50.7 \%\end{array}$ & $19.7 \%$ & $\begin{array}{l}\text { ECG features consistent with } \\
\text { NSTEMI 3.3\% }\end{array}$ & $\begin{array}{l}13.7 \% \\
\text { With cardiac injury 51.2\% }\end{array}$ \\
\hline Shi et al ${ }^{7}$ & $\begin{array}{l}671 \\
52 \%\end{array}$ & $15.8 \%$ & No data & $\begin{array}{l}9.2 \% \\
\text { With cardiac injury } 75.8 \%\end{array}$ \\
\hline Lala et $\mathrm{al}^{8}$ & $\begin{array}{l}2736 \\
40.4 \%\end{array}$ & $\begin{array}{l}36 \% \\
\text { Mild 16.6\% } \\
\text { severe >0.09 ng/dL } \\
19.4 \%\end{array}$ & No data & $\begin{array}{l}18.5 \% \\
\text { Mild troponin elevation } \\
\text { HR } 1.75 \\
\text { severe HR } 3.03\end{array}$ \\
\hline
\end{tabular}

Abbreviations: ACS, acute coronary syndrome; HR, hazard ratio; MI, myocardia infarction; NSTEMI, non-ST segment elevation myocardial infarction.

reported in a case series ${ }^{14}$ of 4 patients ( 1 acute and 3 very late). Various case reports of unusual presentations continue to emerge during the pandemic. STEMI mimics in the form of dynamic ST elevations have been reported. ST elevations are predominantly seen in inferior leads. In two of these reports, a diagnosis of myocarditis was entertained in view of constellation of findings, elevated troponins, decrease in ejection fraction, and absence of obstructive coronary artery disease. In a third case report, ${ }^{15}$ a 29 -year-old morbidly obese, mechanically ventilated patient developed ST elevation in inferior leads, with normal troponin, absence of wall motion abnormalities, and normal ejection fraction on echocardiogram. He was managed conservatively, and his ST-segment changes resolved and were attributed to acute severe right ventricular strain due to acute respiratory distress syndrome. In another report, ${ }^{16}$ a 42 -year-old man with history of single episode of fever 2 weeks prior presented with STEMI. Angiography revealed left anterior descending (LAD) artery and right coronary artery (RCA) occlusion for which he underwent PCI. In view of multivessel occlusion in a low-risk young patient, SARS-CoV-2 infection was confirmed by reverse transcriptase polymerase chain reaction. STEMI could have been precipitated due to a prothrombotic milieu induced by a viral infection. This case report highlights the need for testing for SARS-CoV-2 in all STEMI patients in the setting of a pandemic, especially in unusual presentations. In another case report, ${ }^{17}$ a young woman who was in close contact with COVID-19 patient presented with inferior STEMI, and angiography revealed normal epicardial vessels. SARS-CoV-2 infection was confirmed and she received antiretroviral therapy. She also received inotropes and mechanical ventilation. Repeat ECG revealed diffuse ST elevation and echocardiogram suggested severe LV dysfunction. She had normal white and red cell counts, mild acute kidney and liver injury, and surprisingly only slightly increased high-sensitivity C-reactive protein (hsCRP) $(1.4 \mathrm{mg} / \mathrm{dl})$ but markedly elevated troponin I at admission. After deterioration, she had platelet depletion with increased prothrombin time (PTT), international normalized ratio (INR) and marked increase of plasminogen activator inhibitor (PAI-1). Despite immunosuppressive therapy and immunoglobulin, the patient succumbed. Autopsy revealed microthrombi in inferior wall of right and left ventricles. Interestingly, the autopsy showed no evidence of viral infection in any part of heart, possibly due to low overall expression of ACE-2 receptors in heart.

Both American College of Cardiology (ACC) and European Society of Cardiology (ESC) guidelines recommend carefully weighing the balance between healthcare provider's exposure and patient benefit when considering intervention. All efforts should be made to differentiate between type 2 myocardial infarctions and myocarditis versus type 1 myocardial infarctions, and a strategy of fibrinolysis is detrimental, as a significant proportion of patients have nonobstructive CAD. Although fibrinolysis was suggested as the preferred approach in China, American and European studies endorse the role of primary PCI in PCI-capable hospitals factoring in COVID-19 delays. ${ }^{18}$ The Indian strategy, which revolves around fibrinolysis, should be continued in non $\mathrm{PCI}$ capable hospitals and should be used judiciously, taking into account a constellation of findings which include patient presentation and rapid antigen testing before treatment. Cardiological Society of India (CSI) suggested the use of clinical screening, which may be combined with rapid antigen tests, as they become widely available across the country to rule out myocarditis and noncoronary myocardial injury and divide STEMI patients into confirmed COVID-19, suspected COVID-19 and low probability/non COVID-19. In community transmission stage, both confirmed and suspected COVID-19 should be managed similarly, with primary $\mathrm{PCl}$ being preferred option in PCI-capable hospitals and fibrinolysis if there are logistic problems and in non-PCI hospitals. Primary PCI is the preferred strategy in hemodynamically unstable patients, especially if the COVID-19 pneumonia is mild-to-moderate. Rescue PCI is indicated in cases of failed thrombolysis or for a delayed presentation beyond 12 hours, especially if pneumonia is mild-to-moderate. Patients with low probability/non COVID-19 should be managed with PCI following universal precautions as per CSI guidelines. ${ }^{19}$

\section{Myocarditis and Pericarditis}

Cardiac manifestations of COVID-19 like myocarditis and pericarditis were documented in single patient case reports. ${ }^{20-22}$ Hu et al reported a 37-year-old man who presented with chest pain, dyspnea, and diarrhea, and he had 
thousand-fold elevation of cardiac troponin T and NT-proBNP. His LV ejection fraction (LVEF) was markedly decreased to $27 \%$ with normal coronaries on CT angiography. He received intravenous (IV) immunoglobulin and methylprednisolone and supportive care with vasopressors. He was diagnosed to have fulminant myocarditis, as his cardiac biomarkers decreased and LV function improved in this report. ${ }^{20}$

In a case report, ${ }^{21}$ a 53 -year-old woman with SARSCoV-2 infection, along with fatigue, ECG changes, and elevated cardiac biomarkers, had diffuse hypokinesia with decreased LVEF of $40 \%$. Cardiac MRI revealed diffuse biventricular hypokinesia and diffuse late gadolinium enhancement. Her CRP was normal, and she did not have respiratory failure. She received antivirals and high-dose steroids (IV methylprednisolone $1 \mathrm{mg} / \mathrm{kg} /$ day for 3 days). LV function improved partially by the sixth day. In a case report of a 69-year-old woman who presented with COVID-19 disease and cardiogenic shock, endomyocardial biopsy showed Coronavirus particles within the interstitial cells of the myocardium..$^{23}$ In another report, a 43-year-old woman with COVID-19 disease presented with ST elevation and mild LV dysfunction. Endomyocardial biopsy revealed diffuse $\mathrm{T}$ lymphocytic inflammatory infiltrates, interstitial edema, and limited foci of necrosis. SARS$\mathrm{CoV}-2$ virus was not detected within the myocardium. ${ }^{24}$ In a retrospective study of 112 patients in Wuhan, China, 12.5\% presented with abnormalities similar to myocarditis, which is defined as three-fold elevation in cardiac troponin I along with abnormalities on echocardiography and/or electrocardiogram. ${ }^{25} \mathrm{~A}$ cardiovascular magnetic resonance (CMR)-based study of unselected cohort of 100 patients who recently recovered from COVID-19 infection showed 78\% have cardiac involvement, with the most prevalent abnormality being myocardial inflammation in 60\% cases, followed by regional (scar 32\%) and pericardial enhancement (22\%)

\section{Heart Failure}

Heart failure and cardiogenic shock are associated with morbidity and mortality in COVID-19 disease apart from respiratory failure and other organ failure. Potential causes of myocardial dysfunction include type 1 and type 2 myocardial infarction and myocarditis. Nonischemic heart failure is precipitated by cytokine storm syndrome, which is corroborated by markedly elevated inflammatory markers like IL-6, D-dimer, LDH, ferritin, and CRP in patients with severe COVID-19 disease. ${ }^{27,28}$ Patients with high NT-proBNP values $(>88.64 \mathrm{pg} /$ $\mathrm{mL}$ ) had a significantly increased risk of death; therefore, it was included as an independent predictor of mortality. ${ }^{29}$ From across two Chinese hospitals, a study of 191 patients with COVID-19 disease revealed that $23 \%$ had a clinical diagnosis of heart failure. The mortality was $52 \%$ in those with heart failure. ${ }^{5}$ Another retrospective case series of 150 patients with COVID-19 showed that 33\% of deaths were attributed to respiratory failure plus heart failure; additional $7 \%$ had heart failure without respiratory failure. ${ }^{30}$ Case reports of the use of mechanical circulatory support (venoarterial extracorporeal membrane oxygenation [ECMO]) in patients with cardiogenic shock from COVID-19 were documented. ${ }^{31}$
Takotsubo cardiomyopathy (TTC) is characterized by acute and transient regional LV systolic dysfunction and has been reported in respiratory failure and infections. ${ }^{32}$ Women account for $75 \%$ of cases. In an analysis of 118 consecutive laboratory-confirmed COVID-19 patients $^{33}$ who underwent a clinically indicated TTE, 5 (4.2\%) had features consistent with TTC. The median age of patients with TTC was 66 years. Paradoxically, all patients in this series were male. Higher peak levels of troponin and CK-MB were found in patients with TTC myocardial injury than non-TTC myocardial injury, whereas inflammatory and prothrombotic biomarkers were higher among patients with non-TTC myocardial injury. The authors deduced that TTC may be another phenotype of myocardial injury in COVID-19 and should be considered in patients with myocardial injury and new LV dysfunction.

Although sex disaggregated data ${ }^{34}$ from 26 countries suggest higher case fatality rate in men, paradoxically, the case fatality rate in Indian women is higher, that is, 3.3\% of infected women died of the disease compared with $2.9 \%$ of men. ${ }^{35}$ The possible reasons for sex differences in mortality could be the higher incidence of comorbidities in men and high-risk behaviors like smoking in men. As the $\mathrm{X}$ chromosome harbors immune-related genes, women mount stronger innate and adaptive immune responses than men. ${ }^{36}$ This results in faster clearance of pathogens but paradoxically leads to a stronger inflammatory response. Another biologically plausible explanation postulated is that as ACE-2 gene is located on the $\mathrm{X}$ chromosome, women have higher ACE-2 levels and are protected against more severe disease compared with men. ${ }^{37}$ In summary, although the incidence of myocardial injury is equal in men and women, only further investigation into the association of ACE-2 enzyme activity with severe myocardial injury can shed light on sex differences in disease susceptibility and outcomes. Evaluation of hormonal, immunologic, inflammatory, and phenotypic differences in myriad presentations like myocarditis, acute myocardial infarction, and heart failure in COVID-19 is warranted for better understanding of the disease and development of therapeutic targets.

\section{Conflicts of Interest}

None declared

\section{References}

1 Xiong TY, Redwood S, Prendergast B, Chen M. Coronaviruses and the cardiovascular system: acute and long-term implications. Eur Heart J 2020;41(19):1798-1800

2 Li B, Yang J, Zhao F, et al. Prevalence and impact of cardiovascular metabolic diseases on COVID-19 in China. Clin Res Cardiol 2020;109(5):531-538

3 Atri D, Siddiqi HK, Lang J, et al. COVID-19 for the cardiologist: a current review of the virology, clinical epidemiology, cardiac and other clinical manifestations and potential therapeutic strategies. JACC Basic Transl Sci 2020 (e-pub ahead of print). doi: https://doi.org/10.1016/j.jacbts.2020.04.002

$4 \mathrm{Wu}, \mathrm{M}$, Huang S, Liu J, et al Association between comorbidities and the risk of death in patients with COVID-19: sex-specific differences. medRxiv2020 doi: https://doi.org/1 0.1101/2020.05.22.20109579 
5 Zhou F, Yu T, Du R, et al. Clinical course and risk factors for mortality of adult inpatients with COVID-19 in Wuhan, China: a retrospective cohort study. Lancet 2020;395(10229): 1054-1062

6 Shi S, Qin M, Shen B, et al. Association of cardiac injury with mortality in hospitalized patients with COVID-19 in Wuhan, China. JAMA Cardiol 2020 (e-pub ahead of print). doi: 10.1001/ jamacardio.2020.0950

7 Shaobo S, Qin M, Cai Y,. et al. Characteristics and clinical significance of myocardial injury in patients with severe coronavirus disease 2019. Eur Heart J 2020;41:2070-2079

8 Lala A, Johnson KW, Januzzi JL, et al; Mount Sinai COVID Informatics Center. Prevalence and impact of myocardial injury in patients hospitalized with COVID-19 infection. J Am Coll Cardiol 2020;76(5):533-546

9 Mohanan PP, Mathew R, Harikrishnan S, et al; Kerala ACS Registry Investigators. Presentation, management, and outcomes of 25748 acute coronary syndrome admissions in Kerala, India: results from the Kerala ACS Registry. Eur Heart J 2013;34(2):121-129

10 Bangalore S, Sharma A, Slotwiner A, et al. ST-Segment Elevation in Patients with Covid-19 - A Case Series. N Engl J Med 2020;382(25):2478-2480

11 Acute Coronary Syndromes in COVID-19: Treatment Considerations. Available at: https://www.youtube. com/embed/WYx4xOn97aE?rel=0\&autoplay=1. Accessed September 17, 2020

12 Stefanini GG, ST-elevation myocardial infarction in patients with COVID-19: clinical and angiographic outcomes. Circulation 2020 (e-pub ahead of print). doi: 10.1161/ CIRCULATIONAHA.120.047525

13 Hamadeh A, Aldujeli A, Briedis K, et al. Characteristics and outcomes in patients presenting with COVID-19 and ST-segment elevation myocardial infarction. Am J Cardiol 2020;131:1-6

14 Prieto-Lobato A, A case series of stent thrombosis during the COVID-19 pandemic. J Am Coll Cardiol 2020 (e-pub ahead of print). doi:10.1016/j.jaccas.2020.05.024

15 Loghin C, Chauhan S, Lawless SM. Pseudo-acute myocardial infarction in a young COVID-19 patient. J Am Coll Cardiol 2020 (e-pub ahead of print). doi: https://doi.org/10.1016/j. jaccas.2020.04.015

16 Zendjebil S, Zeitouni M, Batonga M, et al. Acute multi-vessel coronary occlusion revealing COVID-19 in a young adult. J Am Coll Cardiol 2020 (e-pub ahead of print). doi: https://dx.doi. org/10.1016\%2Fj.jaccas.2020.06.002

17 Guagliumi G, Sonzogni A, Pescetelli I, Pellegrini D, Finn AV. Microthrombi and ST-segment elevation myocardial infarction in COVID-19. Circulation 2020;142(8):804-809

18 ESC Guidance for the Diagnosis and Management of CV Disease during the COVID-19 pandemic. Available at: https:// escardio.org/Education/COVID-19. Accessed September 17, 2020

19 Kerkar PG, Naik N, Alexander T, et al. Cardiological Society of India: document on acute MI care during COVID-19. Indian Heart Journal 2020 (e-pub ahead of print). doi: https://doi. org/10.1016/j.ihj.2020.04.009

$20 \mathrm{Hu} \mathrm{H}$, Ma F, Wei X, et al. Coronavirus fulminant myocarditis saved with glucocorticoid and human immunoglobulin. Eur Heart J2020 (e-pub ahead of print). doi: https://doi. org/10.1093/eurheartj/ehaa190
21 Inciardi RM, Lupi L, Zaccone G, et al. Cardiac involvement in a patient with coronavirus disease 2019 (COVID-19) JAMA Cardiol 2020 (e-pub ahead of print). doi: 10.1001/ jamacardio.2020.1096

22 Zeng J, Liu Y-X, Yuan J, et al. First case of COVID-19 infection with fulminant myocarditis complication: case report and insights. Infection2020 (e-pub ahead of print). doi: https:// dx.doi.org/10.1007\%2Fs15010-020-01424-5

23 Tavazzi G, Pellegrini C, Maurelli M, et al. Myocardial localization of coronavirus in COVID-19 cardiogenic shock. Eur J Heart Fail 2020;22(5):911-915

24 Sala S, Peretto G, Gramegna M, et al. Acute myocarditis presenting as a reverse Tako-Tsubo syndrome in a patient with SARS-CoV-2 respiratory infection. Eur Heart J 2020; 41(19):1861-1862

25 Deng Q. Hu B, Zhang Y,. et al. Suspected myocardial injury in patients with COVID-19: evidence from front-line clinical observation in Wuhan, China. Int J Cardiol 2020 (e-pub ahead of print). doi: 10.1016/j.ijcard.2020.03.087

26 Valentina O, Puntmann, M. Carerj L, et al Outcomes of cardiovascular magnetic resonance imaging patients recently recovered from corona virus disease2019 (COVID-19). JAMA Cardiol 2020 (e-pub ahead of print). doi: 10.1001/ jamacardio.2020.3557

27 Siddiqi HK, Mehra MR, COVID-19 illness in native and immunosuppressed states: a clinical-therapeutic staging proposal. J Heart Lung Transplant 2020 (e-pub ahead of print). doi: https://dx.doi.org/10.1016\%2Fj.healun.2020.03.012

28 Wang D, Hu B, Hu C, et al. Clinical characteristics of 138 hospitalized patients with 2019 novel coronavirus-infected pneumonia in Wuhan. China. JAMA 2020; 323(11):1061-9

29 Gao L, Jiang D, Wen XS, et al. Prognostic value of NT-proBNP in patients with severe COVID-19. Respir Res 2020;21(1):83

30 Ruan Q Yang K, Wang W, et al. Clinical predictors of mortality due to COVID-19 based on an analysis of data of 150 patients from Wuhan. China: Intensive Care Medicine; 2020

31 Fried JA, Ramasubbu K, Bhatt R, et al. The variety of cardiovascular presentations of COVID-19. Circulation 2020;141(23):1930-1936

32 Medina de Chazal H, Del Buono MG, Keyser-Marcus L, et al. Stress cardiomyopathy diagnosis and treatment: JACC state-of-the-art review. J Am Coll Cardiol 2018;72(16):1955-1971

33 Giustino, G, Croft LB, Oates CP, et al Takotsubo Cardiomyopathy in COVID-19. J Am Coll Cardiol2020 (e-pub ahead of print). doi: $10.1016 /$ j.jacc.2020.05.068

34 Global Health 5050 COVID-19 Sex-Disaggregated Data Tracker. Available at: http://globalhealth5050.org/covid19. Accessed September 17, 2020

35 Joe W, Kumar A, Rajpal S, Mishra US, Subramanian SV. Equal risk, unequal burden? Gender differentials in COVID-19 mortality in India. J Glob Health Sci2020 (e-pub ahead of print). doi: https://doi.org/10.35500/jghs.2020.2.e17

36 Schurz H, Salie M, Tromp G, Hoal EG, Kinnear CJ, Möller M. The $\mathrm{X}$ chromosome and sex-specific effects in infectious disease susceptibility. Hum Genomics 2019;13(1):2

37 Bhatia K, Zimmerman MA, Sullivan JC. Sex differences in angiotensin-converting enzyme modulation of Ang (1-7) levels in normotensive WKY rats. Am J Hypertens 2013;26(5):591-598 